\title{
Suicidality and COVID-19: Suicidal ideation, suicidal behaviors and completed suicides amidst the COVID-19 pandemic (Review)
}

\author{
VASILIKI EFSTATHIOU ${ }^{1}$, MARIA-IOANNA STEFANOU ${ }^{2}$, NIKOLAOS SIAFAKAS ${ }^{3}$, \\ MICHAEL MAKRIS $^{4}$, GEORGIOS TSIVGOULIS ${ }^{2,5}$, VASSILIOS ZOUMPOURLIS ${ }^{6}$, \\ DEMETRIOS A. SPANDIDOS ${ }^{7}$, NIKOLAOS SMYRNIS ${ }^{1}$ and EMMANOUIL RIZOS ${ }^{1}$
}

\author{
${ }^{1}$ Second Department of Psychiatry; ${ }^{2}$ Second Department of Neurology; ${ }^{3}$ Clinical Microbiology Laboratory; \\ ${ }^{4}$ Allergy Unit, Medical School, National and Kapodistrian University of Athens, 'Attikon' University General Hospital, \\ Athens 12462, Greece; ${ }^{5}$ Department of Neurology, University of Tennessee Health Science Center, Memphis, TN 38163, USA; \\ ${ }^{6}$ Biomedical Applications Unit, Institute of Chemical Biology, National Hellenic Research Foundation (NHRF), \\ Athens 11635; ${ }^{7}$ Laboratory of Clinical Virology, Medical School, University of Crete, Heraklion 71003, Greece
}

Received October 19, 2021; Accepted November 22, 2021

DOI: $10.3892 / \mathrm{etm} .2021 .11030$

\begin{abstract}
Since the outbreak of the coronavirus 2019 (COVID-19) pandemic, there has been widespread concern that social isolation, financial stress, depression, limited or variable access to health care services and other pandemic-related stressors may contribute to an increase in suicidal behaviors. In patients who have recovered from COVID-19, an increased risk of developing suicidal behaviors may be noted, while post-COVID syndrome comprises another potential risk factor contributing to increased suicidal behaviors. Despite the initial alarming predictions for an increase in suicide rates due to the COVID-19 pandemic, the majority of published studies to date suggest that experienced difficulties and distress do not inevitably translate into an increased number of suicide-related deaths, at least not in the short-term. Nevertheless, the long-term mental health effects of the COVID-19 pandemic have yet to be unfolded and are likely to remain for a long period of time. Suicide prevention and measures aiming at promoting well-being and mitigating the effects of COVID-19 on mental health, particularly among vulnerable groups, should thus be a priority for healthcare professionals and policymakers amidst the evolving COVID-19 pandemic.
\end{abstract}

Correspondence to: Professor Emmanouil Rizos, Second Department of Psychiatry, Medical School, National and Kapodistrian University of Athens, 'Attikon' University General Hospital, Rimini 1, Chaidari, Athens 12462, Greece

E-mail: erizos@med.uoa.gr

Key words: COVID-19, SARS-CoV-2, suicidality, suicidal behavior, COVID-19 survivors

\section{Contents}

1. Introduction

2. Suicidal behaviors in the context of the COVID-19 pandemic

3. Conclusion

\section{Introduction}

The outbreak of coronavirus 2019 (COVID-19) was declared by the World Health Organization (WHO) as a global public health emergency on January 30, 2020 (1). Currently, worldwide, there have been $>249$ million confirmed cases of COVID-19, and $>5$ million deaths from COVID-19 reported, according to the WHO (2).

From a public health perspective, the current pandemic has underscored that a multitude of predisposing factors may contribute to the rapid spread of severe acute respiratory syndrome coronavirus 2 (SARS-CoV-2), particularly among vulnerable individuals. First, environmental pollution and chronic exposure to chemicals, such as fossil fuel derivatives, as well as exposure to particle matters, metals, and ultraviolet (UV)-B and ionizing radiation have been acknowledged as risk factors for immunodeficiency, that may contribute to the manifestation of COVID-19 (3). Second, a number of studies have underlined that distinct cellular mechanisms may be associated with an increased predisposition to COVID-19, including the dysfunction of high mobility group proteins (HMGBs), which belong to a superfamily of nuclear proteins with DNA-binding properties that have been implicated in immunological, pro-thrombotic and viral replicating cascades (4). Third, there is an increasing understanding of the central role of immunological processes, including aberrant inflammatory responses and cytokine release during the initial stages of viral infection, as well a growing awareness of the pivotal role of humoral and cellular responses in the elimination or conversely, the propagation of COVID-19 (5). 
Of note, the COVID-19 pandemic is considered the most severe pandemic of the 21st century with significant repercussions not only on physical health, but on mental health as well (6-8). It is thus unsurprising, that the development of effective vaccines against SARS-CoV-2 became a priority for the public health agenda from the time of the outbreak of the pandemic. Currently, there are several available vaccines against COVID-19, which have been proven both safe and effective in preventing severe SARS-CoV-2 infection or mortality from COVID-19, while to date, $>50 \%$ of the world population has received at least one dose of a COVID-19 vaccine (9-17). Although causal treatments for COVID-19 remain unavailable, certain beneficial effects from vitamin supplements, probiotics and minerals have been reported in a number of studies, although their role is considered mostly supportive for the immune system (10,18-20).

Multiple public health interventions have also been implemented worldwide to decrease the transmission of SARS-CoV-2 (21). Nevertheless, measures aiming to attenuate the viral spread, such as self-isolation, quarantine, lockdown and movement restrictions, have led to significant restrictions in daily activities and routines, and along with the threat posed by the pandemic, these measures have propagated feelings of fear, uncertainty, stigmatization, social isolation and loneliness (22-24). Furthermore, there have been reports of a significant increase in alcohol consumption and a significant exacerbation of domestic violence during the implementation of the aforementioned restriction measures $(25,26)$.

Thus, there is a growing concern currently regarding the mental health sequelae of the COVID-19 pandemic $(23,27,28)$. In particular, accumulating evidence has been presented of relapses in individuals with pre-existing mental health issues, who are at risk of further deterioration, new-onset mental illness, increased morbidity or even death, while individuals with no prior psychiatric history are also at risk of developing psychological issues $(29,30)$.

Moreover, an increasing number of studies have suggested that patients with COVID-19 may experience neuropsychiatric manifestations, both during the acute and post-illness stages (i.e., COVID-19 survivors) (31-38). The severity of acute SARS-CoV-2 infection appears to be related to subsequent psychiatric or neurological sequelae, while there may be a tendency towards symptom improvement over time (33).

\section{Suicidal behaviors in the context of the COVID-19 pandemic}

Since the outbreak of the COVID-19 pandemic, there has been widespread concern regarding its effect on suicidality, as COVID-19 may increase the risk of individuals developing suicidal behaviors through its effects on a number of well-established suicide risk factors (Fig. 1) $(39,40)$.

Several parameters, both at the population and individual levels, modulate suicide risk (41). Factors that are related to an increased number of suicides, include a history of suicide attempts, mental health issues, experiencing social isolation, facing financial stress or professional difficulties, criminal or legal issues, having a serious physical illness, substance use disorder, adverse childhood experiences, a family history of suicide, having experienced sexual violence, barriers to health care, stigma associated with mental illness or help-seeking, easy access to lethal means and unsafe media portrayals of suicide (42).

It should be noted that during previous infectious disease outbreaks, suicide prevalence appeared to be elevated. Specifically, suicide rates were found to have increased during the bubonic plague, during the Spanish Flu of 1918-1919 in the United States, during the SARS outbreak in China among the elderly, women and lower socio-economic class, as well as during the Ebola infection in Africa (43-46).

Mental, behavioral, financial and psychosocial issues associated with the COVID-19 pandemic may contribute to an increase in suicidal behaviors $(39,40)$. Furthermore, symptoms of psychiatric, neurological and physical illnesses, as well as inflammatory damage to the brain among patients with post-COVID syndrome may be associated with an increased risk of suicidal tendencies in this population, while COVID-19 survivors without post-COVID syndrome may also face such a risk (47).

Suicidal ideation and suicide attempts amidst the COVID-19 pandemic. Suicidal ideation during the COVID-19 pandemic has been associated with loneliness, anxiety, depression, insomnia, impaired family functioning, a history of mental health issues, alcohol misuse, COVID-19-related stress symptoms, concerns over COVID-19, having tested positive for COVID-19, a younger age, an unmarried or divorced marital status, living alone, being a military veteran, previous homelessness, financial strain, housing instability, unemployment, a poor perceived quality of physical health, disability and living with an individual with frail health (48-53). On the other hand, increased social support, resilience, positive feelings regarding the lockdown measures and faith in a supreme being have been identified as significant protective factors against suicidal ideation $(48,49,54)$.

In a previous meta-analysis of 54 studies, including data from 308,596 individuals, that investigated suicide behaviors during the COVID-19 pandemic, increased event rates were found when considered against event rates from pre-pandemic studies (39). Specifically, that study demonstrated during the COVID-19 pandemic, the combined samples' event rate was $10.81 \%$ for suicidal ideation, $4.68 \%$ for suicide attempts and $9.63 \%$ for self-harm, whereas when community, clinical and frontline samples were examined separately, a trend for higher rates was found in the clinical sample. Nevertheless, it should be noted that the majority of the studies were cross-sectional or retrospective chart reviews (39).

A previous longitudinal study examining the trajectory of mental health during the first 6 weeks of lockdown (from March 31 to May 11, 2020) in a UK adult population $(n=3,077)$ revealed increased rates of suicidal ideation during the initial weeks of the pandemic-related lockdown, particularly among young adults (55). On the other hand, a Spanish longitudinal study aiming at examining the suicidal ideation prevalence among 1,103 adults after the pandemic-related lockdown measures were lifted in Spain (May 21, 2020 to June 30, 2020) compared to the pre-lockdown period (June 17, 2019 to March 14, 2020) found no differences regarding the suicidal ideation rates (54).

As regards suicidal ideation during the pandemic, a longitudinal study from Greece in a community sample of 811 adults, investigated suicidal ideation rates during the first (April 7 


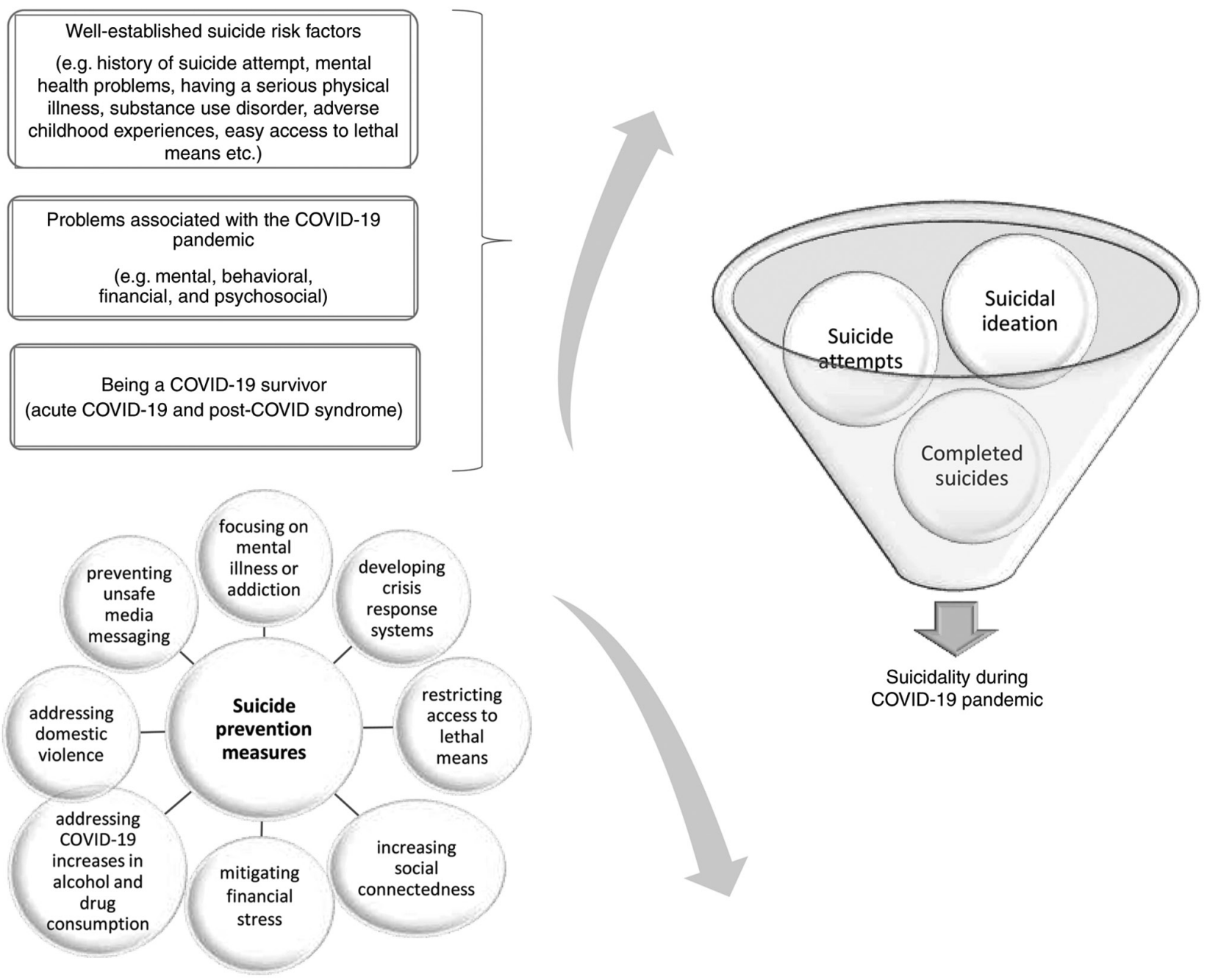

Figure 1. Risk factors for suicidality during COVID-19 pandemic and suicide prevention strategies.

to May 3, 2020) as compared to the second (November 22 to December 21, 2020) pandemic-related national lockdown, and found no differences between the two time periods; depression emerged as the only significant prognostic factor for suicidal ideation incidence (i.e., among individuals who experienced suicidal thoughts during the second, but not the first lockdown) (50). Nevertheless, Veldhuis et al (56), using a global longitudinal convenience sample $(n=1,567)$, surveyed both in April and September, 2020, reported a significant increase over time in suicidal thoughts and behaviors from baseline levels [mean $(M)=2.6$; standard error $(S E)=0.72]$ to the 5-month follow-up $(\mathrm{M}=4.89 ; \mathrm{SE}=0.91 ; \mathrm{P}<0.05)$.

In another study, temporal trends in primary care-recorded self-harm monthly counts (from January, 2019 to May, 2021) were examined using a time series analysis of electronic healthcare records for 2.8 million patients in the North West of England (Greater Manchester conurbation). According to the results of that study, an initial marked reduction in overall self-harm was identified in April, 2020, while a sustained decrease was also observed to the end of May, 2021. The greatest overall reductions were observed during the first national lockdown, while longer-term reductions were largest among males and individuals residing in the most deprived areas; however, an increase emerged in the reports of self-harm among adolescents (10-17 years of age) (57)

A time-trend study in Denmark using health records from hospitals and emergency medical services (covering $46 \%$ of the Danish population), compared the number of psychiatric in-patients, referrals to mental health services and suicidal behavior in the years prior to the COVID-19 pandemic to levels during the first lockdown (March 11 to May 17, 2020), the inter-lockdown period (May 18 to December 15, 2020) and the second lockdown (December 16, 2020 to February 28, 2021). The study did not identify any significant differences with respect to suicidal behavior or referral rates. However, during the pandemic, the rates of psychiatric in-patients decreased compared with the pre-pandemic levels, with the largest decrease being recorded during the first lockdown (58).

Accordingly, a retrospective cross-sectional study in a large, integrated, community-based health system in the USA, on suicide-related encounters in the emergency department demonstrated that the overall rates within 2020 did not increase, and at times the rates decreased, in comparison to those in 2019; however, an increase was observed in 2020 for patients with a substance use disorders (59).

Of note, apart from the psychosocial aspects of suicidality amidst the COVID-19 pandemic, SARS-CoV-2 infection 


\section{Neurobiological correlates of COVID-19 suicidal behavior}

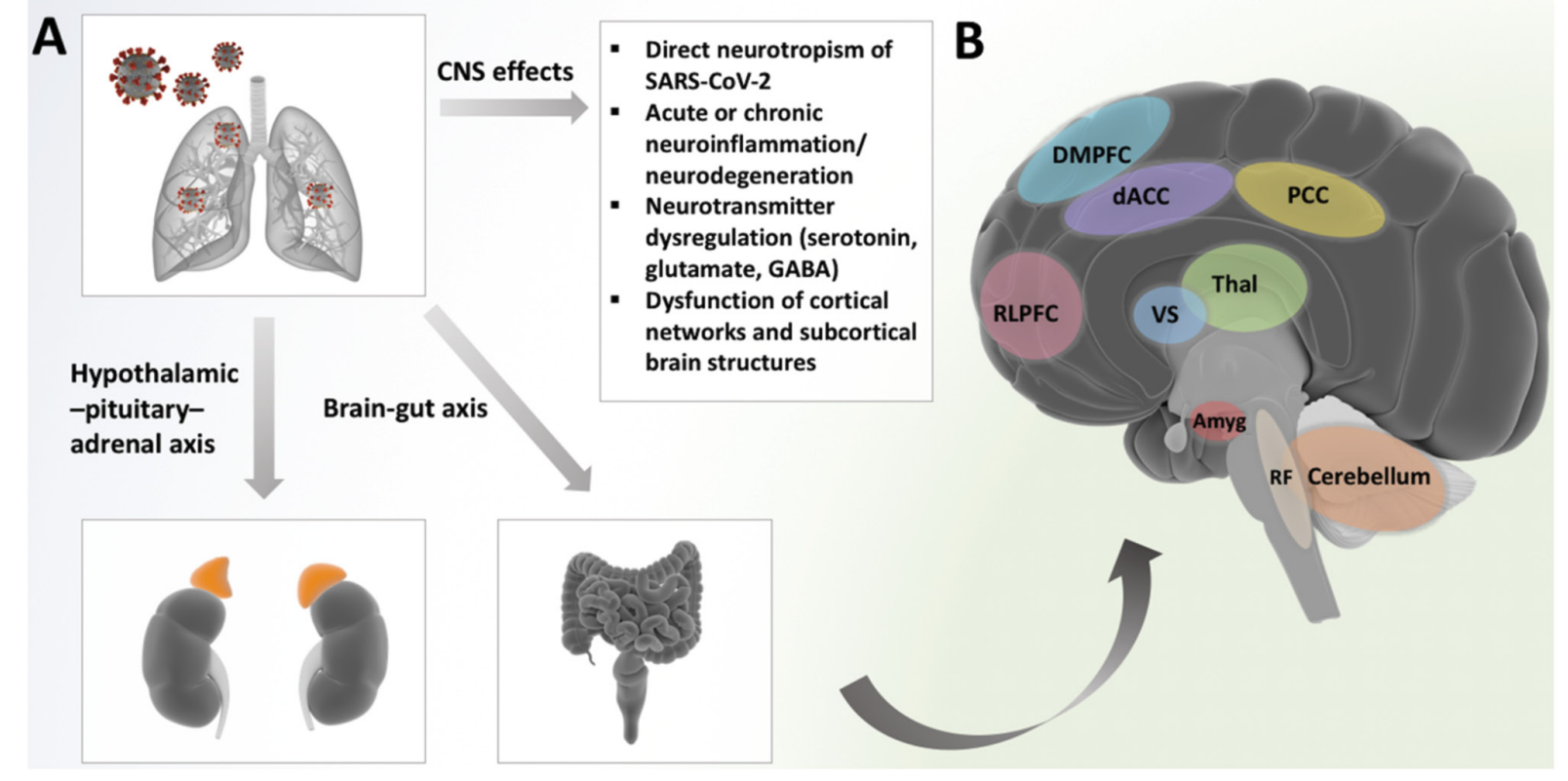

Figure 2. Neurobiological correlates of COVID-19 suicidal behavior. (A) SARS-CoV-2 infection induces distinctive effects in the CNS, which are mediated by the retrograde axonal transport of the virus in the CNS in acute COVID-19, or immune responses, including the cytokine 'storm', that precipitate acute or chronic neuroinflammatory and neurodegenerative cascades. Additionally, SARS-CoV-2 may cause the dysregulation of neurotransmitters (i.e., serotonin, glutamate, GABA) and the dysfunction of large cortical networks and subcortical brain structures, which may be linked to an increased risk of suicidality in patients with COVID-19. Further pathways possibly involved in COVID-19-related suicidal behavior involve the hypothalamic-pituitary adrenal axis. In COVID-19, stress responses and cortisol hypersecretion have been shown to upregulate the hypothalamus-pituitary-adrenal axis (adrenal glands are depicted in orange), while further suggested mechanisms that may contribute to emergence or exacerbation of suicidal behavior involve dysregulation of the gut microbiota and the brain-gut axis. (B) All the aforementioned mechanisms may induce dysfunction in several brain regions, which have been implicated in the pathophysiology of suicidal behavior, and are mainly located in the ventral and dorsal prefrontal cortex, insula, mesial temporal, subcortical and posterior regions. Notably, neuroimaging and histopathological evidence of COVID-19-related suicidality is currently unavailable; thus, the depicted brain regions have been linked with suicidal behavior in previous neuroimaging studies in non-COVID-19 patients with suicidal ideation or behavior. Further brain structures implicated in suicidality include the DMPFC, the hippocampus and the basal ganglia (not shown). Notably, as previously demonstrated, autopsies of patients with COVID-19 have indicated neuronal loss and hypoxic changes in some of the aforementioned brain regions (64). CNS, central nervous system; GABA, glutamate and gamma-aminobutyric acid; RLPFC, rostrolateral prefrontal cortex; DMPFC, dorsomedial prefrontal cortex; dACC, dorsal anterior cingulate cortex; PCC, posterior cingulate cortex; Thal, thalamus; VS, ventral striatum; Amyg, amygdala; RF, reticular formation.

per se may confer an increased risk of suicidal ideation and behaviors. Although the pathophysiological underpinnings of COVID-19 associated suicidality remain to date poorly elucidated, putative mechanisms have been proposed that may increase suicidal vulnerability and the overall risk of suicidal acts in infected patients (60). Accordingly, several mechanisms may be implicated in infection-associated brain damage as follows: The direct neurotropic effects of SARS-CoV-2 mediated by the retrograde axonal transport of the virus from the respiratory mucosa, hypoxic brain injury, peripheral inflammation and monocyte-macrophage system activation that precipitate neuronal dysfunction, and the disruption of the blood-brain barrier that induces a prolonged state of neuroinflammation and possibly neurodegeneration (60-64). The SARS-CoV-2-induced cytokine 'storm' has been shown to play a major role in the propagation of neuropsychiatric symptoms associated with COVID-19, while aberrations in inflammatory cytokines have been proposed as pathophysiological correlates of suicidality $(22,63,65,66)$. In addition, neurotransmitter imbalances, including GABAergic, glutamatergic and serotoninergic pathways, as well as widespread neuronal network dysfunction, have been suggested to provide neurobiological links to COVID-19 suicidality. Moreover, COVID-19 related sleep disturbances, neuroendocrine changes, including cortisol hypersecretion and the dysregulation of the hypothalamus-pituitary-adrenal axis, as well as the dysregulation of the gut microbiota and the brain-gut axis may all contribute to the emergence or exacerbation of suicidal behaviors in patients with COVID-19 (Fig. 2) (22,67-69). Nevertheless, SARS-CoV-2-induced neurobiological changes that precipitate suicidality are possibly intertwined with superimposed psychological stressors associated with COVID-19. Thus, further research is warranted to provide neuroimaging and histopathological data from patients with COVID-19 with suicidal tendencies or behaviors.

Completed suicides amidst the COVID-19 pandemic. From the onset of the COVID-19 pandemic, several mental health experts have expressed concerns regarding a possible increase in the rates of suicide and have even warned of a 'suicide epidemic triggered by COVID-19', a 'dual pandemic of suicide and COVID-19', or a 'suicide mortality and COVID-19-a perfect storm' (43,70-72).

Worldwide, cases of individuals who died through suicide have been reported, mainly in the media, linking their deaths to the fear of COVID-19 infection, fear of transmitting SARS-CoV-2 to others, anxiety over COVID-19, fear of isolation due to imposed country pandemic-related lockdown and 
a lack of knowledge. Further triggers that have been identified for suicides related to the COVID-19 pandemic are xenophobia, COVID-19-related stigma and social boycott, financial insecurities and uncertainty concerning the future $(73,74)$. In previous a review article on suicide reports, possible factors and predictors of COVID-19-related suicide were categorized according to 'social isolation and distancing', 'worldwide lockdown creating economic recession', 'stress, anxiety and pressure in medical healthcare professionals' and 'social boycott and discrimination' (73).

Pirkis et al (75) investigated suicidal trends in the early months of the COVID-19 pandemic (from April 1 to July 31,2020 ) by using an interrupted time-series analysis of preliminary data from 21 countries, including national data from 10 countries and data from 25 regions across a further 11 countries. Specifically, real-time data were acquired on suicides for Australia, Austria, Canada, Chile, Croatia, United Kingdom (England), Estonia, Germany, Italy, Japan, Netherlands, New Zealand, Poland, South Korea, Spain, USA, Brazil, Ecuador, Mexico, Peru and Russia. Their study demonstrated that suicide rates remained unaltered or declined in the early months of the pandemic compared with the expected levels based on the pre-pandemic period, in high-income and upper-middle-income countries (75).

Furthermore, other studies using official data and taking into consideration the effect of temporal trends, have also reported no increase in suicide rates during the early months of the pandemic. In particular, studies from Greece, Australia (Queensland) and the USA (Massachusetts) have demonstrated stability in suicide rates, whereas a study from Peru reported a decline in suicides following the implementation of a pandemic-related lockdown (76-79). Another study from Japan demonstrated fluctuations in suicide rates and highlighted their association with pandemic-induced employment shocks (80).

The absence of an increase in suicide figures, at least during the early months of the pandemic, can be connected to a range of factors. From the beginning of the pandemic, its profound mental health consequences were highlighted. This may have led to governments responding rapidly in order to strengthen existing mental health services, while developing telemedicine services, as well as to enact fiscal support initiatives with the aim of moderating the economic repercussions of pandemic-related lockdowns. Furthermore, for some individuals, the stay-at-home period may have offered them the opportunity to avoid everyday life stresses, while the collective feeling of all individuals facing a pandemic may have been beneficial and helped them feel connected and supported $(75,81)$. Nevertheless, there is evidence to indicate that while suicide rates remain stable or decline during times of acute crises, delayed increases may appear in the period that follows. Indeed, a lag effect of exposure to distressing situations on suicide rates has been previously observed for major world events, such as World War I, World War II and other natural disasters; therefore, a timely implementation of effective prevention strategies is required (81-83).

There is research evidence to support this pattern of an increase in suicides. Following an initial reduction in Japan, where monthly suicide rates decreased by $14 \%$ early stages of the pandemic (February to June, 2020), a subsequent increase in these rates was observed during the second wave (July to October, 2020) by $16 \%$, with a larger increase among females, children and adolescents (84). In addition, while a first report on the short-term effects of the COVID-19 pandemic on suicides, including data from Mexico City, had suggested no increase in the respective rates following the declaration of a national emergency, a second report, following the initial analysis and employing an interrupted time-series analysis indicated that the number of monthly suicides increased during the first 8 months of the pandemic; however, most of the increase occurred beyond June, 2020, and concerned males regardless of their age (85).

Moreover, it is worth noting, that each suicide may affect 5-80 individuals, including nuclear family members, relatives, friends and acquaintances, which are known as 'suicide survivors'. Suicide survivors face an increased risk of prolonged or persistent complex bereavement (with rates reaching up to $40 \%$ ), depression, anxiety and substance-abuse disorders, as well as suicidal behaviors, while social stigma and feelings of shame may discourage them from seeking mental health-related assistance $(74,86)$. The suicide of a 'significant other' amidst the COVID-19 pandemic is even more challenging for suicide survivors, who are already struggling to adjust to new conditions and are likely experiencing psychological difficulties. Feelings of confusion, doubt, non-acceptance, hopelessness, helplessness and stigmatization may emerge, while the fact that death and grief rituals, that would facilitate adjustment to bereavement and a healthy adaptation to the loss, have either been suspended or modified due to pandemic-related regulations, rendering it even harder for suicide survivors to mourn their loved ones and 'say their goodbyes' (74,87).

Suicide prevention during the COVID-19 pandemic is a global priority and requires addressing not only pandemic-specific suicide risk factors, but also pre-pandemic ones. Interventions proposed to significantly mitigate the negative impact of the pandemic on the risk of increased suicidal behaviors include focusing on the needs of individuals with mental illness or addiction, increasing social connectedness, developing robust, accessible and culturally inclusive crisis response systems, restricting access to lethal means, addressing COVID-19-related increases in alcohol consumption and drug overdoses, mitigating financial stress, addressing domestic violence issues and preventing unsafe media and entertainment messaging (Fig. 1) (40).

\section{Conclusion}

The present review has illustrated the impact of the various aspects related to the COVID-19 pandemic on suicidal behaviors, while presenting a comprehensive overview of epidemiological data, as well as evidence on pathophysiological mechanisms implicated in suicidal ideation and behaviors in patients infected with SARS-CoV-2. Improving the current understanding of risk factors that contribute to suicidality is important as it may help mental health professionals in identifying patients at high risk, and can eventually facilitate the development of targeted interventions and prevention strategies.

Despite the initial alarming predictions and warnings regarding an 'epidemic' of suicides due to COVID-19, the 
majority of studies presented in the present review article suggest that difficulties and experienced distress related to the pandemic do not inevitably translate into increased suicide-related deaths, at least not in the short-term. However, it should be pointed out that, even if suicide figures have remained at mostly stable levels, they represent 'real lives lost, real families devastated' (88). Moreover, the long-term mental health effects of the COVID-19 pandemic have yet to be unfolded and are likely to remain for a long period of time or even appear after the actual pandemic. Thus, long-term data are urgently required to reflect the possible long-term repercussions or late-effects of the pandemic. The limitations of the present review thus include the scarcity of available data on long-term outcomes of patients who recovered from acute COVID-19, but continue to suffer from major psychological effects of the pandemic. Future research is urgently required to enable the characterization of risk factors and the early identification of predictors of suicidal behavior during the ongoing pandemic of COVID-19.

Suicide prevention and measures in order to promote mental health and well-being, as well as to mitigate COVID-19 psychological consequences, particularly among vulnerable groups and populations at a higher risk, should be a priority for health care professionals and policymakers.

\section{Acknowledgements}

Not applicable.

\section{Funding}

No funding was received.

\section{Availability of data and materials}

Not applicable.

\section{Authors' contributions}

VE, MIS, NSi and MM wrote the original draft, edited and critically revised the manuscript. GT, VZ, DAS, NSm and ER critically revised and edited the manuscript. All authors substantially contributed to the conception, writing and revision of the work and have read and approved the final manuscript. Data authentication is not applicable.

\section{Ethics approval and consent to participate}

Not applicable.

\section{Patient consent for publication}

Not applicable.

\section{Competing interests}

DAS is the Editor-in-Chief for the journal, but had no personal involvement in the reviewing process, or any influence in terms of adjudicating on the final decision, for this article. The other authors declare that they have no competing interests.

\section{References}

1. Sohrabi C, Alsafi Z, O'Neill N, Khan M, Kerwan A, Al-Jabir A, Iosifidis $\mathrm{C}$ and Agha R: World Health Organization declares global emergency: A review of the 2019 novel coronavirus (COVID-19). Int J Surg 76: 71-76, 2020.

2. World Health Organization (WHO): Coronavirus disease 2019 (COVID-2019): Weekly epidemiological update, 2021. WHO, Geneva, 2021. www.who.int/emergencies/diseases/novel-coronavirus-2019/situation-reports. Accessed November 13, 2021.]

3. Tsatsakis A, Petrakis D, Nikolouzakis TK, Docea AO, Calina D, Vinceti M, Goumenou M,Kostoff RN, Mamoulakis C, Aschner M and Hernández AF: COVID-19, an opportunity to reevaluate the correlation between long-term effects of anthropogenic pollutants on viral epidemic/pandemic events and prevalence. Food Chem Toxicol 141: 111418, 2020.

4. Islam M, Hossen M, Kamaz Z, Zali A, Kumar M, Anca Oana D, Arsene A, Calina D and Sharifi-Rad J: The role of HMGB1 in the immune response to SARS-COV-2 infection: From pathogenesis towards a new potential therapeutic target. Farmacia 69: 621-634, 2021.

5. Neagu M, Calina D, Docea AO, Constantin C, Filippini T, Vinceti M, Drakoulis N, Poulas K, Nikolouzakis TK, Spandidos DA and Tsatsakis A: Back to basics in COVID-19: Antigens and antibodies-Completing the puzzle. J Cell Mol Med 25: 4523-4533, 2021.

6. Leaune E, Samuel M, Oh H, Poulet E and Brunelin J: Suicidal behaviors and ideation during emerging viral disease outbreaks before the COVID-19 pandemic: A systematic rapid review. Prev Med 141: 106264, 2020

7. Kahil K, Cheaito MA, El Hayek R, Nofal M, El Halabi S, Kudva KG, Pereira-Sanchez V and El Hayek S: Suicide during COVID-19 and other major international respiratory outbreaks: A systematic review. Asian J Psychiatr 56: 102509, 2021.

8. Zoumpourlis V, Goulielmaki M, Rizos E, Baliou S and Spandidos DA: [Comment] The COVID-19 pandemic as a scientific and social challenge in the 21st century. Mol Med Rep 22: 3035-3048, 2020.

9. Calina D, Hartung T, Docea AO, Spandidos DA, Egorov AM, Shtilman MI, Carvalho F and Tsatsakis A: COVID-19 vaccines: Ethical framework concerning human challenge studies. Daru 28: 807-812, 2020.

10. Torequl Islam M, Nasiruddin M, Khan IN, Mishra SK, Kudrat-E-Zahan M, Alam Riaz T, Ali ES, Rahman MS, Mubarak MS, Martorell M, et al: A perspective on emerging therapeutic interventions for COVID-19. Front Public Health 8: $281,2020$.

11. Calina D, Hernández AF, Hartung T, Egorov AM, Izotov BN, Nikolouzakis TK, Tsatsakis A, Vlachoyiannopoulos PG and Docea AO: Challenges and scientific prospects of the newest generation of mRNA-Based vaccines against SARS-CoV-2. Life (Basel) 11: 907, 2021.

12. Kostoff RN, Kanduc D, Porter AL, Shoenfeld Y, Calina D, Briggs MB, Spandidos DA and Tsatsakis A: Vaccine- and natural infection-induced mechanisms that could modulate vaccine safety. Toxicol Rep 7: 1448-1458, 2020.

13. Calina D, Docea AO, Petrakis D, Egorov AM, Ishmukhametov AA, Gabibov AG, Shtilman MI, Kostoff R, Carvalho F, Vinceti M, et al: Towards effective COVID-19 vaccines: Updates, perspectives and challenges (Review). Int J Mol Med 46: 3-16, 2020.

14. Calina D, Sarkar C, Arsene AL, Salehi B, Docea AO, Mondal M, Islam MT,Zali A and Sharifi-Rad J: Recent advances, approaches and challenges in targeting pathways for potential COVID-19 vaccines development. Immunol Res 68: 315-324, 2020.

15. Hernández AF, Calina D, Poulas K, Docea AO and Tsatsakis AM: Safety of COVID-19 vaccines administered in the EU: Should we be concerned? Toxicol Rep 8: 871-879, 2021.

16. KostoffRN,CalinaD,KanducD,Briggs MB, VlachoyiannopoulosP, Svistunov AA and Tsatsakis A: Why are we vaccinating children against COVID-19? Toxicol Rep 8: 1665-1684, 2021.

17. Ritchie H, Mathieu E, Rodés-Guirao L, Appel C, Giattino C, Ortiz-Ospina E, Hasell J, Macdonald B, Beltekian D and Roser M: Coronavirus pandemic (COVID-19). OurWorldInData.org, 2020. https://ourworldindata.org/covid-vaccinations. Accessed November 13, 2021.]

18. Islam M, Salehi B, Karampelas O, Sharifi-Rad J, Anca Oana D, Martorell M and Calina D: High skin melanin content, vitamin D deficiency and immunity: Potential interference for severity of COVID-19. Farmacia 68: 970-983, 2020. 
19. Sharifi-Rad J, Rodrigues CF, Stojanović-Radić Z, Dimitrijević M, Aleksić A, Neffe-Skocińska K, Zielińska D, Kołożyn-Krajewska D, Salehi B, Milton Prabu S, et al: Probiotics: Versatile bioactive components in promoting human health. Medicina (Kaunas) 56: 433, 2020

20. Islam MT, Quispe C, Martorell M, Docea AO, Salehi B, Calina D, Reiner Ž and Sharifi-Rad J: Dietary supplements, vitamins and minerals as potential interventions against viruses: Perspectives for COVID-19. Int J Vitam Nutr Res: Jan 13, 2021 (Epub ahead of print).

21. Ayouni I, Maatoug J, Dhouib W, Zammit N, Fredj SB, Ghammam R and Ghannem H: Effective public health measures to mitigate the spread of COVID-19: A systematic review. BMC Public Health 21: 1015, 2021.

22. Dhrisya C, Prasathkumar M, Becky R, Anisha S, Sadhasivam S Essa MM, Chidambaram SB, Al-Balushi B, Guillemin GJ and Qoronfleh MW: Social and biological parameters involved in suicide ideation during the COVID-19 pandemic: A narrative review. Int J Tryptophan Res 13: 1178646920978243, 2020.

23. Serafini G, Parmigiani B, Amerio A, Aguglia A, Sher L and Amore M: The psychological impact of COVID-19 on the mental health in the general population. QJM 113: 531-537, 2020

24. Tsamakis K, Triantafyllis AS, Tsiptsios D, Spartalis E, Mueller C, Tsamakis C, Chaidou S, Spandidos DA, Fotis L, Economou M, et al: COVID-19 related stress exacerbates common physical and mental pathologies and affects treatment (Review). Exp Ther Med 20: 159-162, 2020.

25. Kumar A and Nayar KR: COVID 19 and its mental health consequences. J Mental Health 30: 1-2, 2021

26. Calina D, Hartung T, Mardare I, Mitroi M, Poulas K, Tsatsakis A, Rogoveanu I and Docea AO: COVID-19 pandemic and alcohol consumption: Impacts and interconnections. Toxicol Rep 8: 529-535, 2021

27. Duan L and Zhu G: Psychological interventions for people affected by the COVID-19 epidemic. Lancet Psychiatry 7 : 300-302, 2020

28. Tsamakis K, Tsiptsios D, Ouranidis A, Mueller C, Schizas D, Terniotis C, Nikolakakis N, Tyros G, Kympouropoulos S, Lazaris A, et al: COVID-19 and its consequences on mental health (Review). Exp Ther Med 21: 244, 2021.

29. Byrne A, Barber R and Lim CH: Impact of the COVID-19 pandemic-a mental health service perspective. Prog Neurol Psychiatry 25: 27-33b, 2021.

30. Vai B, Mazza MG, Delli Colli C, Foiselle M, Allen B, Benedetti F, Borsini A, Casanova Dias M, Tamouza R, Leboyer M, et al: Mental disorders and risk of COVID-19-related mortality, hospitalisation, and intensive care unit admission: A systematic review and meta-analysis. Lancet Psychiatry 8: 797-812, 2021.

31. Dinakaran D, Manjunatha N, Naveen Kumar C and Suresh BM: Neuropsychiatric aspects of COVID-19 pandemic: A selective review. Asian J Psychiatr 53: 102188, 2020.

32. Mazza MG, De Lorenzo R, Conte C, Poletti S, Vai B, Bollettini I Melloni EMT, Furlan R, Ciceri F, Rovere-Querini P, et al: Anxiety and depression in COVID-19 survivors: Role of inflammatory and clinical predictors. Brain Behav Immun 89: 594-600, 2020.

33. Schou TM, Joca S, Wegener G and Bay-Richter C: Psychiatric and neuropsychiatric sequelae of COVID-19-A systematic review. Brain Behav Immun 97: 328-348, 2021.

34. Kumar S, Veldhuis A and Malhotra T: Neuropsychiatric and cognitive sequelae of COVID-19. Front Psychology 12: 577529, 2021.

35. Giannopoulou I, Galinaki S, Kollintza E, Adamaki M, Kympouropoulos S, Alevyzakis E, Tsamakis K, Tsangaris I, Spandidos DA, Siafakas N, et al: COVID-19 and post-traumatic stress disorder: The perfect 'storm' for mental health (Review). Exp Ther Med 22: 1162, 2021.

36. Tsatsakis A, Calina D, Falzone L, Petrakis D, Mitrut R, Siokas V, Pennisi M, Lanza G, Libra M, Doukas SG, et al: SARS-CoV-2 pathophysiology and its clinical implications: An integrative overview of the pharmacotherapeutic management of COVID-19. Food Chem Toxicol 146: 111769, 2020.

37. Huang C, Huang L, Wang Y, Li X, Ren L, Gu X, Kang L, Guo L, Liu M, Zhou X, et al: 6-month consequences of COVID-19 in patients discharged from hospital: A cohort study. Lancet 397: 220-232, 2021.

38. Taquet M, Geddes JR, Husain M, Luciano S and Harrison PJ: 6-month neurological and psychiatric outcomes in 236379 survivors of COVID-19: A retrospective cohort study using electronic health records. Lancet Psychiatry 8: 416-427, 2021.
39. Dubé JP, Smith MM, Sherry SB, Hewitt PL and Stewart SH: Suicide behaviors during the COVID-19 pandemic: A meta-analysis of 54 studies. Psychiatry Res 301: 113998, 2021.

40. Moutier C: Suicide prevention in the COVID-19 Era: Transforming threat into opportunity. JAMA Psychiatry 78 : 433-438, 2021.

41. Turecki G and Brent DA: Suicide and suicidal behaviour. Lancet 387: 1227-1239, 2016.

42. Centers for Disease Control and Prevention (CDC): Suicide Prevention-Risk and Protective Factors. CDC, Atlanta, GA, 2021. www.cdc.gov/suicide/factors/index.html. Accessed October 2, 2021.

43. Banerjee D, Kosagisharaf JR and Sathyanarayana Rao TS: 'The dual pandemic' of suicide and COVID-19: A biopsychosocial narrative of risks and prevention. Psychiatry Res 295: 113577, 2021.

44. Wasserman IM: The impact of epidemic, war, prohibition and media on suicide: United States, 1910-1920. Suicide Life Threat Behav 22: 240-254, 1992

45. Cheung YT, Chau PH and Yip PS: A revisit on older adults suicides and Severe Acute Respiratory Syndrome (SARS) epidemic in Hong Kong. Int J Geriatr Psychiatry 23: 1231-1238, 2008.

46. Bitanihirwe BK: Monitoring and managing mental health in the wake of Ebola. Commentary. Ann Ist Super Sanita 52: 320-322, 2016.

47. Sher L: Post-COVID syndrome and suicide risk. QJM 114: 95-98, 2021.

48. Elbogen EB, Lanier M, Blakey SM, Wagner HR and Tsai J: Suicidal ideation and thoughts of self-harm during the COVID-19 pandemic: The role of COVID-19-related stress, social isolation, and financial strain. Depress Anxiety 38: 739-748, 2021.

49. Papadopoulou A,Efstathiou V, Yotsidi V,Pomini V, Michopoulos I, Markopoulou E, Papadopoulou M, Tsigkaropoulou E, Kalemi G, Tournikioti K, et al: Suicidal ideation during COVID-19 lockdown in Greece: Prevalence in the community, risk and protective factors. Psychiatry Res 297: 113713, 2021.

50. Efstathiou V, Michopoulos I, Yotsidi V, Smyrnis N, Zompola C, Papadopoulou A, Pomini V, Papadopoulou M, Tsigkaropoulou E, Tsivgoulis G, et al: Does suicidal ideation increase during the second COVID-19 lockdown? Psychiatry Res 301: 113990, 2021.

51. Antonelli-Salgado T, Monteiro GMC, Marcon G, Roza TH, Zimerman A, Hoffmann MS, Cao B, Hauck S, Brunoni AR and Passos IC: Loneliness, but not social distancing, is associated with the incidence of suicidal ideation during the COVID-19 outbreak: A longitudinal study. J Affect Disord 290: 52-60, 2021.

52. Killgore WDS, Cloonan SA, Taylor EC, Fernandez F, Grandner MA and Dailey NS: Suicidal ideation during the COVID-19 pandemic: The role of insomnia. Psychiatry Res 290: 113134,2020

53. Killgore WDS, Cloonan SA, Taylor EC and Dailey NS: Loneliness: A signature mental health concern in the era of COVID-19. Psychiatry Res 290: 113117, 2020.

54. Ayuso-Mateos JL, Morillo D, Haro JM, Olaya B, Lara E and Miret M: Changes in depression and suicidal ideation under severe lockdown restrictions during the first wave of the COVID-19 pandemic in Spain: A longitudinal study in the general population. Epidemiol Psychiatr Sci 30: e49, 2021.

55. O'Connor RC, Wetherall K, Cleare S, McClelland H, Melson AJ, Niedzwiedz CL, O'Carroll RE, O'Connor DB, Platt S, Scowcroft E, et al: Mental health and well-being during the COVID-19 pandemic: Longitudinal analyses of adults in the UK COVID-19 Mental Health \& Wellbeing study. Br J Psychiatry: Oct 21, 2020 (Epub ahead of print).

56. Veldhuis CB, Nesoff ED, McKowen ALW, Rice DR, Ghoneima H, Wootton AR, Papautsky EL, Arigo D, Goldberg S and Anderson JC: Addressing the critical need for long-term mental health data during the COVID-19 pandemic: Changes in mental health from April to September 2020. Prev Med 146: 106465, 2021.

57. Steeg S, Bojanić L, Tilston G, Williams R, Jenkins DA, Carr MJ Peek N, Ashcroft DM, Kapur N, Voorhees J and Webb RT: Temporal trends in primary care-recorded self-harm during and beyond the first year of the COVID-19 pandemic: Time series analysis of electronic healthcare records for 2.8 million patients in the Greater Manchester Care Record. EClinicalMedicine 41: 101175, 2021.

58. Rømer TB, Christensen RHB, Blomberg SN, Folke F, Christensen HC and Benros ME: Psychiatric admissions, referrals, and suicidal behavior before and during the COVID-19 pandemic in denmark: A time-trend study. Acta Psychiatr Scand 144: 553-562, 2021 
59. Ridout KK, Alavi M, Ridout SJ, Koshy MT, Awsare S, Harris B Vinson DR, Weisner CM, Sterling S and Iturralde E: Adult Suicide-Related emergency department encounters during the COVID-19 Pandemic: A Cross-sectional study. Lancet Regional Health Am 4: 100078, 2021.

60. Conejero I, Nobile B, Olié E and Courtet P: How does COVID-19 affect the neurobiology of suicide? Curr Psychiatry Rep 23: 16, 2021.

61. Troyer EA, Kohn JN and Hong S: Are we facing a crashing wave of neuropsychiatric sequelae of COVID-19? Neuropsychiatric symptoms and potential immunologic mechanisms. Brain Behav Immun 87: 34-39, 2020

62. Steiner J, Gos T, Bogerts B, Bielau H, Drexhage HA and Bernstein HG: Possible impact of microglial cells and the monocyte-macrophage system on suicidal behavior. CNS Neurol Disord Drug Targets 12: 971-979, 2013.

63. Ngo B, Lapp SA, Siegel B, Patel V, Hussaini L, Bora S, Philbrook B, Weinschenk K, Wright L, Anderson EJ, et al: Cerebrospinal fluid cytokine, chemokine, and SARS-CoV-2 antibody profiles in children with neuropsychiatric symptoms associated with COVID-19. Mult Scler Relat Disord 55: 103169 , 2021.

64. Solomon IH, Normandin E, Bhattacharyya S, Mukerji SS Keller K, Ali AS, Adams G, Hornick JL, Padera RF Jr and Sabeti P: Neuropathological features of Covid-19. N Engl J Med 383: 989-992, 2020.

65. Brundin L, Bryleva EY and Thirtamara Rajamani K: Role of inflammation in suicide: From mechanisms to treatment. Neuropsychopharmacology 42: 271-283, 2017.

66. Schmaal L, van Harmelen AL, Chatzi V, Lippard ETC Toenders YJ, Averill LA, Mazure CM and Blumberg HP: Imaging suicidal thoughts and behaviors: A comprehensive review of 2 decades of neuroimaging studies. Mol Psychiatry 25 : 408-427, 2020

67. Pfennig A, Kunzel HE, Kern N, Ising M, Majer M, Fuchs B Ernst G, Holsboer F and Binder EB: Hypothalamuspituitary-adrenal system regulation and suicidal behavior in depression. Biol Psychiatry 57: 336-342, 2005.

68. Mello MT, Silva A, Guerreiro RC, da-Silva FR, Esteves AM, Poyares D, Piovezan R, Treptow E, Starling M, Rosa DS, et al: Sleep and COVID-19: Considerations about immunity, pathophysiology, and treatment. Sleep Sci 13: 199-209, 2020.

69. Chen J and Vitetta L: Gut-brain axis in the neurological comorbidity of COVID-19. Brain Commun 3: fcab118, 2021.

70. Reger MA, Stanley IH and Joiner TE: Suicide mortality and coronavirus disease 2019-a perfect storm? JAMA Psychiatry 77: 1093-1094, 2020.

71. Tandon R: COVID-19 and suicide: Just the facts. Key learnings and guidance for action. Asian J Psychiatr 60: 102695, 2021.

72. Mannix R, Lee LK and Fleegler EW: Coronavirus disease 2019 (COVID-19) and Firearms in the United States: Will an epidemic of suicide follow? Ann Intern Med 173: 228-229, 2020.

73. Thakur V and Jain A: COVID 2019-suicides: A global psychological pandemic. Brain Behav Immun 88: 952-953, 2020.
74. Pinto S, Soares J, Silva A, Curral R and Coelho R: COVID-19 suicide survivors-a hidden grieving population. Front Psychiatry 11: 626807, 2020.

75. Pirkis J, John A, Shin S, DelPozo-Banos M, Arya V, Analuisa-Aguilar P, Appleby L, Arensman E, Bantjes J, Baran A, et al: Suicide trends in the early months of the COVID-19 pandemic: An interrupted time-series analysis of preliminary data from 21 countries. Lancet Psychiatry 8: 579-588, 2021.

76. Faust JS, Shah SB, Du C, Li S-X, Lin Z and Krumholz HM: Suicide deaths during the COVID-19 Stay-at-Home advisory in massachusetts, March to May 2020. JAMA Netw Open 4: e2034273, 2021.

77. Calderon-Anyosa RJC and Kaufman JS: Impact of COVID-19 lockdown policy on homicide, suicide, and motor vehicle deaths in Peru. Prev Med 143: 106331, 2021.

78. Vandoros S, Theodorikakou O, Katsadoros K, Zafeiropoulou D and Kawachi I: No evidence of increase in suicide in Greece during the first wave of Covid-19. medRxiv: doi: https://doi.org/10. 1101/2020.11.13.20231571.

79. Leske S, Kõlves K, Crompton D, Arensman E and de Leo D: Real-time suicide mortality data from police reports in Queensland, Australia, during the COVID-19 pandemic: An interrupted time-series analysis. Lancet Psychiatry 8: 58-63, 2021.

80. Ando M and Furuichi M: The impact of COVID-19 employment shocks on suicide and poverty alleviation programs: An early-stage investigation. medRxiv: doi: https://doi.org/10.1101/ 2020.11.16.20232850.

81. Wasserman D, Iosue M, Wuestefeld A and Carli V: Adaptation of evidence-based suicide prevention strategies during and after the COVID-19 pandemic. World Psychiatry 19: 294-306, 2020.

82. Zalsman G, Stanley B, Szanto K, Clarke DE, Carli V and Mehlum L: Suicide in the time of COVID-19: Review and recommendations. Arch Suicide Res 24: 477-482, 2020.

83. Lester D: Suicide rates before, during and after the world wars. Eur Psychiatry 9: 262-264, 1994.

84. Tanaka T and Okamoto S: Increase in suicide following an initial decline during the COVID-19 pandemic in Japan. Nat Hum Behav 5: 229-238, 2021.

85. Borges G, Garcia JA, Pirkis J, Spittal MJ and Lopez-Arellano O: Suicide after and during the COVID-19 pandemic in Mexico City. SSRN, Rochester, NY, 2021. https://ssrn.com/abstract=3879041 or http://dx.doi.org/10.2139/ssrn.3879041. Accessed June, 30, 2021.

86. Berman AL: Estimating the population of survivors of suicide: Seeking an evidence base. Suicide Life Threat Behav 41: 110-116, 2011.

87. Castle J and Phillips WL: Grief rituals: Aspects that facilitate adjustment to bereavement. J Loss Trauma 8: 41-71, 2003.

88. Appleby L: What has been the effect of Covid-19 on suicide rates? BMJ 372: n834, 2021.

c) (i) $(-)$ This work is licensed under a Creative Commons

EY NO NO Attribution-NonCommercial-NoDerivatives 4.0 International (CC BY-NC-ND 4.0) License. 